\title{
Efficient Pseudorandom Generators Based on the DDH Assumption
}

\author{
Reza Rezaeian Farashahi ${ }^{1,2}$, Berry Schoenmakers ${ }^{1}$, and Andrey Sidorenko ${ }^{1}$ \\ ${ }^{1}$ Dept. of Mathematics and Computer Science, TU Eindhoven, \\ P.O. Box 513, 5600 MB Eindhoven, The Netherlands \\ ${ }^{2}$ Dept. of Mathematical Sciences, Isfahan University of Technology, \\ P.O. Box 85145 Isfahan, Iran
}

\begin{abstract}
A family of pseudorandom generators based on the decisional Diffie-Hellman assumption is proposed. The new construction is a modified and generalized version of the Dual Elliptic Curve generator proposed by Barker and Kelsey. Although the original Dual Elliptic Curve generator is shown to be insecure, the modified version is provably secure and very efficient in comparison with the other pseudorandom generators based on discrete log assumptions.

Our generator can be based on any group of prime order provided that an additional requirement is met (i.e., there exists an efficiently computable function that in some sense enumerates the elements of the group). Two specific instances are presented. The techniques used to design the instances, for example, the new probabilistic randomness extractor are of independent interest for other applications.
\end{abstract}

\section{Introduction}

A pseudorandom generator is a deterministic algorithm that converts a short sequence of uniformly distributed random bits into a longer sequence of bits that cannot be distinguished from uniformly random by a computationally bounded algorithm. It is known that a pseudorandom generator can be constructed from any one-way function [13. Thus, intractability of the discrete logarithm problem suffices to construct a pseudorandom generator. Such a construction was first proposed by Blum and Micali [2. However, the Blum-Micali pseudorandom generator and similar ones are inefficient in the sense that only a single bit is output per modular exponentiation. In this paper, we show that the stronger assumption that the decisional Diffie-Hellman problem is hard to solve (DDH assumption) gives rise to much more efficient pseudorandom generators.

Using strong assumptions in order to improve performance of cryptographic schemes is a common practice nowadays. In particular, several pseudorandom generators based on strong number theoretic assumptions have been proposed during the last decade. For instance, Patel and Sundaram [25], Gennaro [8] show that efficient pseudorandom generators can be built if one assumes that computing discrete logarithms with short exponents is a hard problem. Steinfeld et al. [29] propose an improved version of the well-known RSA generator assuming the 
intractability of a strong variant of the RSA problem. In comparison with many other assumptions, the DDH assumption is thoroughly studied (for more details about intractability of the DDH problem, refer e.g. to [24]) and has become a basis for a wide variety of cryptographic schemes.

Security of our construction is tightly related to the intractability of the DDH problem.

\subsection{Related Work}

Our work is inspired by the publication of Barker and Kelsey [1, in which the so-called Dual Elliptic Curve generator is proposed. Let $P$ and $Q$ be points on a prime order elliptic curve over a prime field $\mathbb{F}_{p}$ such that $p$ is close to $2^{256}$. Let $q$ denote the order of the curve. On input $s_{0}$ chosen uniformly at random from $\mathbb{Z}_{q}$ the Dual Elliptic Curve generator produces two sequences of points $s_{i} P$ and $s_{i} Q$ such that $s_{i}$ is set to be the $x$-coordinate of $s_{i-1} P, i=1,2, \ldots, k$. The generator outputs $k$ binary strings each string consisting of the 240 least significant bits of the $x$-coordinate of $s_{i} Q$. The sequence of points $s_{i} Q$ is shown to be indistinguishable from the sequence of uniformly random points of the elliptic curve under the assumption that the DDH problem and the non-standard $x$-logarithm problem are intractable in $\mathrm{E}\left(\mathbb{F}_{p}\right)[3$. However, the binary sequence produced by the generator turns out to be distinguishable from uniform. The reason is that points of the elliptic curve are transformed into random bits in an improper way 1027 .

Some ideas of the Dual Elliptic Curve generator are present in the earlier work by Naor and Reingold 24. Let $p$ be a prime and let $g$ be a generator of a subgroup of $\mathbb{Z}_{p}^{*}$ of prime order $q$. Let $a \in \mathbb{Z}_{q}$ be a fixed number. Naor and Reingold 24 propose a simple function $G$ that on input $b \in \mathbb{Z}_{q}$ outputs $\left(g^{b}, g^{a b}\right)$. If $b$ is chosen uniformly at random, the output of the function is computationally indistinguishable from uniform under the DDH assumption in the subgroup. Note, however, that function $G$ produces random elements of the subgroup rather than random bits and therefore it is not a pseudorandom generator in the sense of Definition 1 (converting random elements of the subgroup into random bits is a nontrivial problem). Moreover, although function $G$ doubles the input it cannot be iterated to produce as much pseudorandomness as required by the application. Namely, it is not clear how to produce a new value of $b$ given two group elements $g^{b}$ and $g^{a b}$. Accordingly, the goal of Naor and Reingold [24] is to construct not a pseudorandom generator but a pseudorandom function, for which function $G$ turns out to be a suitable building block.

\subsection{Our Contributions}

We modify and generalize the Dual Elliptic Curve generator such that the modified version is provably secure under the DDH assumption. In comparison with the original Dual Elliptic Curve generator, our generator can be based on any group of prime order meeting an additional requirement (i.e., there exists an efficiently computable function that in some sense enumerates the elements of 
the group). The new generator is more efficient than all known pseudorandom generators based on discrete log assumptions.

We present two specific instances of the new pseudorandom generator.

The first instance is based on the group of quadratic residues modulo a safe prime $p=2 q+1$. This instance uses an elegant idea of Cramer and Shoup [5] who show that there exists a simple bijective function that maps quadratic residues modulo $p$ to $\mathbb{Z}_{q}$.

The second instance is based on an arbitrary prime order subgroup of $\mathbb{Z}_{p}^{*}$, where $p$ is prime but not necessarily a safe prime. To construct this instance, we first propose a surprisingly simple probabilistic randomness extractor that provided with some extra randomness converts a uniformly random element of the subgroup of order $q$ into a uniformly random number in $\mathbb{Z}_{q}$, which in turn can be easily converted into a string of uniformly random bits using, for instance, algorithm $Q_{2}$ from [15] (for an overview of probabilistic randomness extractors, refer to 28]). Note that all (probabilistic and deterministic) extractors known so far can only convert random elements of the subgroup into bits that are statistically close to uniform.

We derive the security parameters of the new pseudorandom generators from the corresponding security reductions. For this purpose, we make practical assumptions about intractability of the discrete logarithm problems in the corresponding groups.

\section{Preliminaries}

In this section, we introduce some conventions and recall basic definitions.

\section{$2.1 \quad$ Notation}

Let $x$ and $y$ be random variables taking on values in a finite set $S$. The statistical distance between $x$ and $y$ is defined as

$$
\Delta(x, y)=\frac{1}{2} \sum_{\alpha \in S}|\operatorname{Pr}[x=\alpha]-\operatorname{Pr}[y=\alpha]| .
$$

We say that algorithm $\mathcal{D}$ distinguishes $x$ and $y$ with advantage $\epsilon$ if and only if

$$
|\operatorname{Pr}[\mathcal{D}(x)=1]-\operatorname{Pr}[\mathcal{D}(y)=1]| \geq \epsilon .
$$

If the statistical distance between $x$ and $y$ is less than $\epsilon$ then no algorithm distinguishes $x$ and $y$ with advantage $\epsilon$ (see, e.g., [20, Exercise 22]).

Throughout, we let $U_{m}$ denote a random variable uniformly distributed on $\mathbb{Z}_{m}$. And, we say that an algorithm is $T$-time if it halts in time at most $T$.

\subsection{Pseudorandom Generators}

Consider a deterministic algorithm PRG : $\{0,1\}^{n} \mapsto\{0,1\}^{M}$, where $M>n$. Loosely speaking, PRG is called a pseudorandom generator if it maps uniformly 
distributed input into an output which is computationally indistinguishable from uniform. The input is called the seed and the output is called the pseudorandom sequence. The precise definition is given below.

A $T$-time algorithm $\mathcal{D}:\{0,1\}^{M} \mapsto\{0,1\}$ is said to be a $(T, \epsilon)$-distinguisher for PRG if

$$
\left|\operatorname{Pr}\left[\mathcal{D}\left(\operatorname{PRG}\left(U_{2^{n}}\right)\right)=1\right]-\operatorname{Pr}\left[\mathcal{D}\left(U_{2^{M}}\right)=1\right]\right| \geq \epsilon
$$

Definition 1 (Pseudorandom generator). Algorithm PRG is called a $(T, \epsilon)$ secure pseudorandom generator if no $(T, \epsilon)$-distinguisher exists for PRG.

An important question is what level of security $(T, \epsilon)$ suffices for practical applications of pseudorandom generators. Unfortunately, the level of security is often chosen arbitrarily. Knuth ([17, p. 176) sets $\epsilon=0.01$ and consider several values for $T$ up to $53.5 \cdot 10^{12}$ Mips-Years]. In [6], the security level is set to $T=1$ Mips-Year and $\epsilon=0.01$. In [8], $T=3.5 \cdot 10^{10}$ Mips-Years, $\epsilon=0.01$.

The fact that a pseudorandom generator is $(T, \epsilon)$-secure does not automatically mean that the generator is $\left(T^{\prime}, \epsilon^{\prime}\right)$-secure for all $T^{\prime}$ and $\epsilon^{\prime}$ such that $T^{\prime} / \epsilon^{\prime} \leq T / \epsilon$. For instance, if a pseudorandom generator is $(T, 0.01)$-secure it does not necessarily mean that the generator is $\left(T^{\prime}, 0.009\right)$-secure even if $T \gg T^{\prime}$. The reason is that a $\left(T^{\prime}, 0.009\right)$-distinguisher cannot always be transformed into a $(T, 0.01)$-distinguisher. Indeed, the only way to improve the success probability of the distinguisher is to run it several times on the same input. However, the latter does not always help since there might be "bad" inputs, that is, inputs for which the success probability of the distinguisher is very low or equals 0 .

It is reasonable to require that a pseudorandom generator is secure for all pairs $(T, \epsilon)$ such that the time-success ratio $T / \epsilon$ is below a certain bound that is set to be $2^{80}$ time units throughout this paper (the time unit is defined in Section 2.4). Time-success ratio is a standard way to define security of cryptographic schemes [2013].

\subsection{Decisional Diffie-Hellman Problem}

Let $\mathbb{G}$ be a multiplicative group of prime order $q$. For $x, y \in \mathbb{G}$ and $s \in \mathbb{Z}_{q}$ such that $y=x^{s}, s$ is called the discrete logarithm of $y$ to the base $x$. We write $s=\log _{x} y$. The discrete logarithm (DL) problem is to find $s$ given $x$ and $y$.

Definition 2 (DDH problem). Let $X_{D D H} \in \mathbb{G}^{4}$ be a random variable uniformly distributed on the set consisting of all 4-tuples $(x, y, v, w) \in \mathbb{G}^{4}$ such that $\log _{x} v=\log _{y} w$ and let $Y_{D D H} \in R \mathbb{G}^{4}$. Algorithm $\mathcal{D}$ is said to solve the decisional Diffie-Hellman (DDH) problem in $\mathbb{G}$ with advantage $\epsilon$ if it distinguishes the random variables $X_{D D H}$ and $Y_{D D H}$ with advantage $\epsilon$, that is,

$$
\left|\operatorname{Pr}\left[\mathcal{D}\left(X_{D D H}\right)=1\right]-\operatorname{Pr}\left[\mathcal{D}\left(Y_{D D H}\right)=1\right]\right| \geq \epsilon .
$$

\footnotetext{
${ }^{1}$ A Mips-Year is defined as the amount of computation that can be performed in one year by a single DEC VAX 11/780 (see also [19]).
} 
Related to the decisional Diffie-Hellman problem is the computational DiffieHellman (CDH) problem (given $x, y$ and $x^{s}$, compute $y^{s}$ ).

Clearly, the DL problem is at least as hard to solve as the CDH problem. The CDH problem is proved to be equivalent to the DL problem under certain conditions 2122]. Moreover, no groups are known such that the CDH problem is strictly easier to solve than the DL problem. The common practice is to assume that these two problems are equally hard.

On the other hand, there exist groups (e.g., $\mathbb{Z}_{p}^{*}$ ) in which a random instance of the $\mathrm{CDH}$ problem is believed to be hard while the DDH problem is easy. The latter groups are referred to as the non-DDH groups 9]. Furthermore, Wolf [30] shows that for all groups $\mathbb{G}$ an algorithm that solves the DDH problem in $\mathbb{G}$ is of no help for solving the $\mathrm{CDH}$ problem in $\mathbb{G}$. However, the computational gap between the $\mathrm{DDH}$ problem and the $\mathrm{CDH}$ problem is difficult to estimate. It is believed that except for the non-DDH groups, there is no way to solve the DDH problem rather than to solve the $\mathrm{CDH}$ problem.

We do not use non-DDH groups in this paper. To compute security parameters for the pseudorandom generators, we assume that the DDH problem and the DL problem are equally hard, in agreement with common practice. We formalize this as follows.

Let $T_{D L}$ be the running time of the best known algorithm for solving a random instance of the DL problem in a group $\mathbb{G}$. Of course, $T_{D L}$ depends on the group $\mathbb{G}$, that is, $T_{D L}=T_{D L}(\mathbb{G})$. For instance, in the case of finite fields, $T_{D L}$ corresponds to the running time of the discrete logarithm variant of the Number Field Sieve, while for most of the ordinary elliptic curves the best known algorithms are the exponential square root attacks.

Assumption 1. Unless $\mathbb{G}$ is a non-DDH group, no $T$-time algorithm solves the $D D H$ problem in $\mathbb{G}$ with probability $\epsilon$ if $T / \epsilon \leq T_{D L}(\mathbb{G})$.

\subsection{Conventions}

Time Units. A unit of time has to be set to measure the running time of the algorithms. Throughout this paper, the unit of time is one DES encryption. According to the data from [19], a software implementation of DES is estimated to take about 360 Pentium clock cycles. Therefore, we assign

1 time unit $=360$ Pentium clock cycles.

Security level. The table by Lenstra and Verheul [19] implies that $2^{80}$ DES encryptions will be infeasible for classical computers until the year 2013. Therefore, we set $2^{80}$ time units as the security level to be reached.

Modular multiplication cost. In [19, it is reported that multiplication modulo $p$ takes about $\left(\log _{2} p\right)^{2} / 24$ Pentium clock cycles, that is,

$$
\left(\log _{2} p\right)^{2} /(24 \cdot 360) \text { time units. }
$$

Complexity of discrete logarithm variant of the NFS. The discrete logarithm variant of the Number Field Sieve (NFS) algorithm solves the discrete logarithm problem in a $n$-bit prime field in expected time $L(n)=$ 
$A \exp \left((1.9229+o(1))(n \ln 2)^{1 / 3}(\ln (n \ln 2))^{2 / 3}\right)$, where $A$ is a constant. Following [19], we assume that the $o(1)$-term is zero and estimate the constant $A$ from experimental data. Unfortunately, practical experience with the discrete logarithm variant of the NFS is limited. On the other hand, there are several data points for the Number Field Sieve factoring algorithm. For instance, factoring a 512-bit integer is reported to take about $3 \cdot 10^{17}$ Pentium clock cycles [19]. Since computing discrete logarithms in $n$-bit fields takes about the same amount of time as factoring $n$-bit integers for any $n$ in the current range of interest (cf. [19]), this suggests that $A \approx 4.7 \cdot 10^{-5}$ and thus

$$
L(n)=4.7 \cdot 10^{-5} \exp \left(1.9229(n \ln 2)^{1 / 3}(\ln (n \ln 2))^{2 / 3}\right) \text { time units. }
$$

It is believed that the discrete logarithm problem in the extension field is as hard as the discrete logarithm problem in the prime field of similar size (cf. [18]).

\section{DDH Generator}

In this section, our main result is presented. We propose a new provably secure pseudorandom generator. We call it the DDH generator, since the security of this generator relies on the intractability of the DDH problem in the corresponding group. In contrast with the Dual Elliptic Curve generator [1, the DDH generator can be based on any group of prime order provided that an additional requirement is met (i.e., there exists an efficiently computable function enum that "enumerates" the elements of the group).

\subsection{Construction of the Generator}

Let $\mathbb{G}$ be a multiplicative group of prime order $q$ and let enum : $\mathbb{G} \times \mathbb{Z}_{l} \mapsto$ $\mathbb{Z}_{q} \times \mathbb{Z}_{l}, l>0$, be a bijection. Thus, on uniformly distributed input, function enum produces uniformly distributed output. Typically, but not necessarily, $l$ is chosen to be small. The advantage of a smaller $l$ is that the seed of the generator is shorter.

Let $x, y \in_{R} \mathbb{G}$. The seed of the DDH generator (Algorithm 1 ) is $s_{0} \in_{R} \mathbb{Z}_{q}$ and randp $_{0}$, randq $_{0} \in R \mathbb{Z}_{l}$. The DDH generator transforms the seed into the sequence of $k$ pseudorandom numbers from $\mathbb{Z}_{q}$.

Note that the random elements $x$ and $y$ are not part of the seed. These two elements are system parameters that are not necessarily kept secret. In the security analysis of the generator we assume that $x$ and $y$ are known to the distinguisher.

\subsection{Security Analysis}

The following theorem implies that under the DDH assumption for group $\mathbb{G}$ an output sequence of the DDH generator is indistinguishable from a sequence of uniformly random numbers in $\mathbb{Z}_{q}$. 


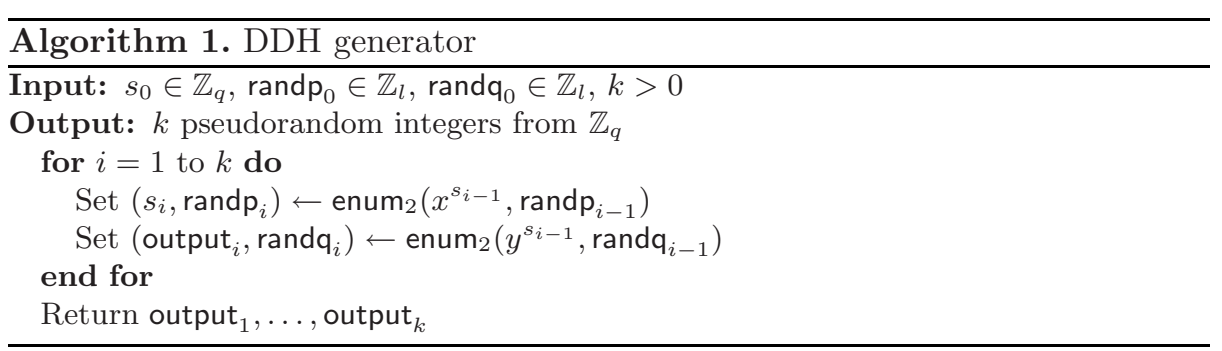

Theorem 2. Suppose there exists a T-time algorithm that distinguishes the output of the DDH generator from the sequence of independent uniformly distributed random numbers in $\mathbb{Z}_{q}$ with advantage $\epsilon$. Then the DDH problem in $\mathbb{G}$ can be solved in time $T$ with advantage $\epsilon / k$.

Proof. Suppose there exists a $T$-time algorithm $\mathcal{D}$ that distinguishes the output of the DDH generator from a sequence of independent uniformly distributed random numbers in $\mathbb{Z}_{q}$ with advantage $\epsilon$, that is,

$$
\mid \operatorname{Pr}\left[\mathcal{D}\left(\text { output }_{1}, \ldots, \text { output }_{k}\right)=1\right]-\operatorname{Pr}[\mathcal{D}(U)=1] \mid \geq \epsilon,
$$

where $U=\left(u_{1}, \ldots, u_{k}\right), u_{i} \in_{R} \mathbb{Z}_{q}, i=1, \ldots, k$. Let $j \in_{R}\{1,2, \ldots, k\}$. Due to the classical hybrid argument (see, e.g., [11, Section 3.2.3]),

$$
\left|\operatorname{Pr}\left[\mathcal{D}\left(Z_{j}\right)=1\right]-\operatorname{Pr}\left[\mathcal{D}\left(Z_{j+1}\right)=1\right]\right| \geq \epsilon / k,
$$

where

$$
\begin{aligned}
Z_{j} & =\left(u_{1}, \ldots, u_{j-1}, \text { output }_{1}, \ldots, \text { output }_{k-j+1}\right), \\
Z_{j+1} & =\left(u_{1}, \ldots, u_{j-1}, u_{j}, \text { output }_{1}, \ldots, \text { output }_{k-j}\right),
\end{aligned}
$$

the probability is taken not only over internal coin flips of $\mathcal{D}$ but also over the choice of $j$. Now, we show how to solve the DDH problem in $\mathbb{G}$ using the distinguisher $\mathcal{D}$ as a building block. Let $(x, y, v, w) \in \mathbb{G}^{4}$. A solver for the DDH problem decides if $\log _{x} v=\log _{y} w$ or $v$ and $w$ are independent uniformly distributed random elements of $\mathbb{G}$ as follows.

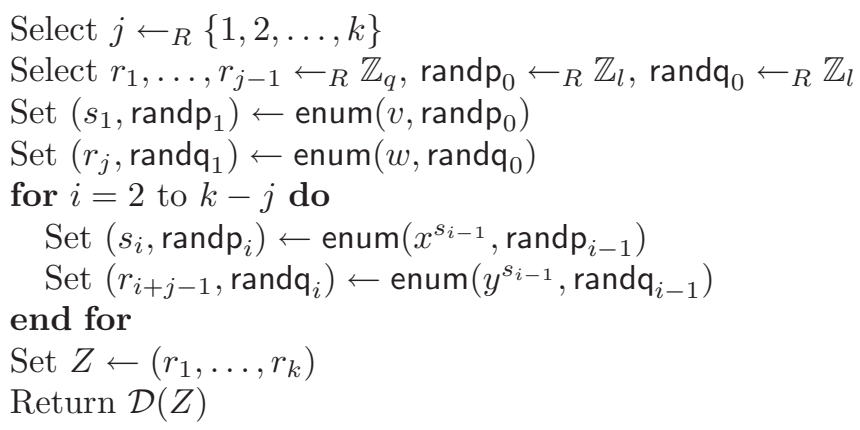


If there exists $s_{0} \in \mathbb{Z}_{q}$ such that $v=x^{s_{0}}$ and $w=y^{s_{0}}$ then $r_{j}$ and $r_{j+1}$ are distributed as the first and the second outputs of the DDH generator respectively, so $Z$ is distributed as $Z_{j}$.

Otherwise, if $v$ and $w$ are independent uniformly distributed random elements of $\mathbb{G}$ then $r_{j+1}$ is distributed as the first output of the DDH generator while $r_{j}$ is uniformly distributed over $\mathbb{Z}_{q}$ and independent of $r_{j+1}$, so $Z$ is distributed as $Z_{j+1}$.

Therefore, the above algorithm solves the DDH problem in $\mathbb{G}$ in time at most $T$ with advantage $\epsilon / k$.

The DDH generator is not a pseudorandom generator in terms of Definition 1, It outputs numbers in $\mathbb{Z}_{q}$ rather than bits. However, converting random numbers into random bits is a relatively easy problem. For instance, one can use Algorithm $\mathrm{Q}_{2}$ from [15], which was presented without analysis. It can actually be shown, however, that Algorithm $\mathrm{Q}_{2}$ produces on average $n-2$ bits given a uniformly distributed random number $U_{q}$, where $n$ denotes the bit length of $q$. In the latter case, the average number of bits produced by the generator is $k(n-2)$.

For the sake of simplicity, throughout this paper, we assume that $q$ is close to a power of 2 , that is, $0 \leq\left(2^{n}-q\right) / 2^{n} \leq \delta$ for a small $\delta$. So, the uniform element $U_{q}$ is statistically close to $n$ uniformly random bits.

The following simple lemma is a well-known result (the proof can be found, for instance, in [4]).

Lemma 1. Under the condition that $0 \leq\left(2^{n}-q\right) / 2^{n} \leq \delta$, the statistical distance between $U_{q}$ and $U_{2^{n}}$ is bounded above by $\delta$.

The next statement implies that if $q$ is close to a power of 2 , the DDH generator is a cryptographically secure pseudorandom generator under the DDH assumption in $\mathbb{G}$.

Corollary 1. Let $0 \leq\left(2^{n}-q\right) / 2^{n} \leq \delta$. Suppose the DDH generator is not $(T, \epsilon)$ secure. Then there exists an algorithm that solves the DDH problem in $\mathbb{G}$ in time at most $T$ with advantage $\epsilon / k-\delta$.

Proof. Suppose there exists a distinguisher $\mathcal{D}:\{0,1\}^{k n} \mapsto\{0,1\}$ that runs in time at most $T$ and

$$
\left.\mid \operatorname{Pr}\left[\mathcal{D} \text { (output }_{1}, \ldots, \text { output }_{k}\right)=1\right]-\operatorname{Pr}\left[\mathcal{D}\left(U_{2^{k n}}\right)=1\right] \mid \geq \epsilon .
$$

Let $u_{i} \in_{R} \mathbb{Z}_{q}, i=1, \ldots, k$, and $U=\left(u_{1}, \ldots, u_{k}\right)$. Lemma 1 implies that the statistical distance $\Delta\left(U, U_{2^{k n}}\right) \leq k \delta$. Thus,

$$
\mid \operatorname{Pr}\left[\mathcal{D}\left(\text { output }_{1}, \ldots, \text { output }_{k}\right)=1\right]-\operatorname{Pr}[\mathcal{D}(U)=1] \mid \geq \epsilon-k \delta .
$$

Now, the statement follows from Theorem 2.

\section{Specific Instances of the DDH Generator}

To implement the DDH generator, one has to choose the group $\mathbb{G}$ of prime order $q$ and function enum that enumerates the group elements. In this section, we propose two specific instances of the DDH generator. 
Throughout this section we assume that $q$ is close to a power of 2 , that is, $0 \leq\left(2^{n}-q\right) / 2^{n} \leq \delta$ for a small $\delta$ and some integer $n$. We like to emphasize that this assumption is made for the sake of simplicity only. $M$ denotes the total number of pseudorandom bits produced by the generator.

\subsection{Group of Quadratic Residues Modulo Safe Prime}

To construct the first instance of the DDH generator, we use an elegant idea of Cramer and Shoup [5] who show that there exists a simple deterministic function that enumerates elements of the group of quadratic residues modulo safe prime $p$.

Let $p$ be a safe prime, $p=2 q+1$, where $q$ is prime. Let $\mathbb{G}_{1}$ be a group of nonzero quadratic residues modulo $p$. The order of $\mathbb{G}_{1}$ equals $q$. Consider the following function enum $1: \mathbb{G}_{1} \mapsto \mathbb{Z}_{q}$,

$$
\text { enum }_{1}(x)=\left\{\begin{array}{l}
x, \text { if } 1 \leq x \leq q \\
p-x, \text { if } q+2 \leq x<p \\
0, \text { otherwise. }
\end{array}\right.
$$

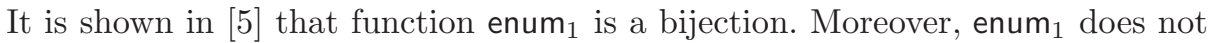
require any additional input, so in terms of Section $3.1 l=1$.

Let $x, y \in \mathbb{G}_{1}$. Let $s_{0} \in_{R} \mathbb{Z}_{q}$ be the seed. Generator $\mathrm{PRG}_{1}$ (Algorithm 4.1) is a deterministic algorithm that transforms the seed into the sequence of $k n$ pseudorandom bits.

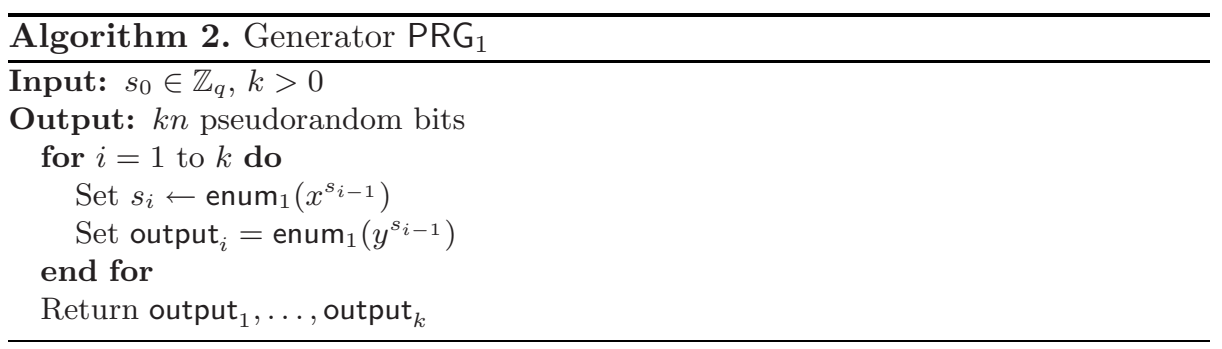

The next statement follows from Corollary 1

Proposition 1. Suppose pseudorandom generator $\mathrm{PRG}_{1}$ is not $(T, \epsilon)$-secure. Then there exists an algorithm that solves the DDH problem in $\mathbb{G}_{1}$ in time at most $T$ with advantage $\epsilon / k-\delta$.

The seed length $n$ plays the role of security parameter of the generator. Clearly, smaller $n$ gives rise to a faster generator. On the other hand, for larger $n$ the generator is more secure. Our goal is to select $n$ as small as possible such that the generator is $(T, \epsilon)$-secure for all $T, \epsilon$ such that $T / \epsilon<2^{80}$ time units.

For $\delta=\epsilon /(2 k)$, the generator is $(T, \epsilon)$-secure if

$$
2 k T / \epsilon<T_{D L}\left(\mathbb{G}_{1}\right)
$$


where $T_{D L}\left(\mathbb{G}_{1}\right)$ is the running time of the fastest known method for solving the discrete logarithm problem in $\mathbb{G}_{1}$. According to the current state of the art, we set $T_{D L}\left(\mathbb{G}_{1}\right)$ to be the running time of the discrete logarithm variant of the Number Field Sieve $L(n)$ (see Section 2.4). Note that $k=M / n$. Then, (2) holds if $2 M T /(n \epsilon)<L(n)$. For $M=2^{20}$ and $T / \epsilon=2^{80}$, the smallest parameter $n$ that satisfies the above inequality is $n \approx 1600$.

Recall that $q$ satisfies $0 \leq\left(2^{n}-q\right) / 2^{n} \leq \delta$. We have assumed that $\delta=\epsilon /(2 k)$. For $M=2^{20}, n=1600$, and $\epsilon=2^{-80}$, this condition implies that $0<2^{n}-q<$ $2^{1500}$. There are plenty of safe primes $p=2 q+1$ such that $0<2^{1600}-q<2^{1500}$.

\subsection{Arbitrary Prime Order Subgroup of $\mathbb{Z}_{p}^{*}$}

In this section, we show that the DDH generator can be based not only on the group of quadratic residues modulo a safe prime but on any prime order subgroup of $\mathbb{Z}_{p}^{*}$, where $p$ is a prime but not necessarily a safe prime.

Let $q$ be a prime factor of $p-1, p-1=l q, l \geq 2$, such that $\operatorname{gcd}(l, q)=1$. If $p$ is a safe prime then $l=2$. Denote by $\mathbb{G}_{2}$ a subgroup of $\mathbb{Z}_{p}^{*}$ of order $q$. Throughout this section, multiplication of integers is done modulo $p$.

Let split $t_{2}: \mathbb{Z}_{p}^{*} \mapsto \mathbb{Z}_{q} \times \mathbb{Z}_{l}$ denote a bijection that splits an element of $\mathbb{Z}_{p}^{*}$ into two smaller numbers. An example of split ${ }_{2}$ is a function that on input $z \in \mathbb{Z}_{p}^{*}$ returns $(z-1) \bmod q$ and $\lfloor(z-1) / q\rfloor$. Let $t \in \mathbb{Z}_{p}^{*}$ be an element of order $l$. Let enum $_{2}: \mathbb{G}_{2} \times \mathbb{Z}_{l} \mapsto \mathbb{Z}_{q} \times \mathbb{Z}_{l}$ be the following function:

$$
\operatorname{enum}_{2}(x, \text { rand })=\operatorname{split}_{2}\left(x t^{\text {rand }}\right),
$$

where $x \in \mathbb{G}_{2}$, rand $\in \mathbb{Z}_{l}$. The following lemma shows that enum 2 is a bijection and thus it is suitable for building the DDH generator.

Lemma 2. Function enum ${ }_{2}$ defined above is a bijection.

Proof. Let $f: \mathbb{G}_{2} \times \mathbb{Z}_{l} \mapsto \mathbb{Z}_{p}^{*}$ be defined as $f(x$, rand $)=x t^{\text {rand }} \bmod p$ for $x \in \mathbb{G}_{2}$ and rand $\in \mathbb{Z}_{l}$. To prove the statement of the lemma, we first show that $f$ is a bijection.

Suppose that $x_{1} t^{\text {rand }_{1}}=x_{2} t^{\text {rand }_{2}}$ for $x_{i} \in \mathbb{G}_{2}$, rand $_{i} \in \mathbb{Z}_{l}, i=1,2$. Since $x_{2} \in$ $\mathbb{G}_{2}, x_{2} \neq 0$. Then, $x_{1} / x_{2}=t^{\text {rand }_{1}-\text { rand }_{2}} \in \mathbb{G}_{2}$, so $t^{q\left(\text { rand }_{1}-\text { rand }_{2}\right)}=1$. Therefore, $l$ divides $q\left(\operatorname{rand}_{1}-\operatorname{rand}_{2}\right)$. Since $\operatorname{gcd}(q, l)=1$, it implies that $l$ divides rand ${ }_{1}-\operatorname{rand}_{2}$. The latter implies that rand $_{1}=\operatorname{rand}_{2}$ and thus $x_{1}=x_{2}$.

Therefore, $f$ is indeed a bijection and thus enum ${ }_{2}$ is also a bijection as a composition of two bijective functions.

Let $\mathrm{PRG}_{2}$ denote the instance of the $\mathrm{DDH}$ generator that uses the group $\mathbb{G}_{2}$ and the function enum ${ }_{2}$ defined above. The next statement follows from Corollary 1

Proposition 2. Suppose pseudorandom generator $\mathrm{PRG}_{2}$ is not $(T, \epsilon)$-secure. Then there exists an algorithm that solves the DDH problem in $\mathbb{G}_{2}$ in time at most $T$ with advantage $\epsilon / k-\delta$. 
Let $m$ denote the bit length of $p$. At each step $i=1, \ldots, n$, pseudorandom generator $\mathrm{PRG}_{2}$ computes $x^{s_{i-1}}$ and $y^{s_{i-1}}$ and then uses these elements to evalu-

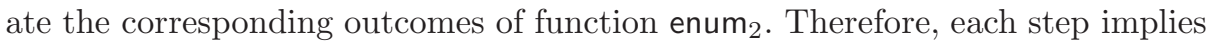
two modular exponentiations with $n$-bit exponents and two modular exponentiations with $(m-n)$-bit exponents. Since $\mathrm{PRG}_{2}$ outputs $n$ bits per step the computational effort per output bit is proportional to $\mathrm{m}^{3} / n$. Our goal is now to determine parameters $m$ and $n$ that minimize the computational effort under the condition that the generator is $(T, \epsilon)$-secure for all $T, \epsilon$ satisfying $T / \epsilon<2^{80}$.

For $\delta=\epsilon /(2 k)$, generator $\mathrm{PRG}_{2}$ is $(T, \epsilon)$-secure if

$$
2 k T / \epsilon<T_{D L}\left(\mathbb{G}_{2}\right),
$$

where $T_{D L}\left(\mathbb{G}_{2}\right)$ is the running time of the fastest known method for solving the discrete logarithm problem in $\mathbb{G}_{2}$. The best algorithms for solving the discrete logarithm problem in $\mathbb{G}_{2}$ are Pollard's rho method in $\mathbb{G}_{2}$ and the discrete logarithm variant of the Number Field Sieve in the full multiplicative group $\mathbb{Z}_{p}^{*}$. The running time of Pollard's rho method is estimated to be $0.88 \sqrt{q}$ group operations (cf. [19]). Since $k=M / n$, condition (3) implies that

$$
2 M T /(n \epsilon)<\min \left[L(m), 0.88 \cdot 2^{n / 2} m^{2} /(24 \cdot 360)\right] .
$$

For $M=2^{20}, T / \epsilon=2^{80}$, the above condition is satisfied for $m \gtrsim 1600, n \gtrsim 160$. The computational effort is minimized if $n \approx m$.

In comparison with $P R G_{1}$, the seed of $P R G_{2}$ is somewhat longer, although if $n \approx m$ it is roughly of the same size. Moreover, $\mathrm{PRG}_{2}$ is less efficient than $\mathrm{PRG}_{1}$

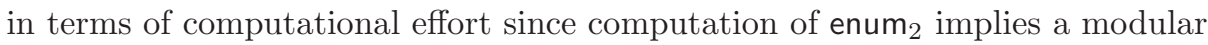

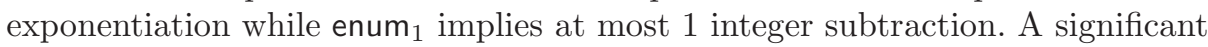
advantage of $P R G_{2}$ versus $P R G_{1}$ is that the former can be based on any prime order subgroup of $\mathbb{Z}_{p}^{*}$ for any prime $p$ provided that the size of the subgroup is sufficiently large to resist Pollard's rho attack.

\subsection{Discussion}

Function enum ${ }_{2}$ used as a building block of generator $\mathrm{PRG}_{2}$ is of independent interest. The reason is that this function can be viewed as a probabilistic randomness extractor (for an overview of probabilistic randomness extractors, refer to [28]). Provided with some extra randomness, it converts a uniformly random element of a subgroup of $\mathbb{Z}_{p}^{*}$ of order $q$ into a uniformly random number in $\mathbb{Z}_{q}$, which in turn can be easily converted into a string of uniformly random bits using, for instance, algorithm $Q_{2}$ from [15]. Note that all (probabilistic and deterministic) extractors known so far can only convert random elements of the subgroup into bits that are statistically close to uniform.

The new extractor can be used not only for designing pseudorandom generators but also for key exchange protocols to convert the random group element shared by the parties involved into the random binary string.

If the size of the subgroup $q$ is sufficiently large, our extractor is more efficient than the general purpose probabilistic randomness extractors (e.g., the universal 
hash functions [13]) in terms of the number of extra random bits required. For instance, if the statistical distance to be reached is $2^{-80}$ our extractor requires less extra randomness than universal hash functions if the size of the subgroup is at least $p / 2^{160}$. If the size of the subgroup is close to the size of the group $p$, our extractor requires just few extra random bits.

The recently proposed deterministic extractor by Fouque et al. [7 does not require any extra randomness to produce the output. However, it extracts substantially less than half of the bits of a uniformly distributed random element of the subgroup. Our extractor does require extra randomness rand $\in \mathbb{Z}_{l}, l \geq 1$, but one gets this randomness back in the sense that the extractor outputs not only the integer from $\mathbb{Z}_{q}$ but also an element of $\mathbb{Z}_{l}$. The crucial advantage of our extractor is that it extracts all the bits of the subgroup element.

\section{Generator PRG 1 Versus Gennaro's Generator}

In this section, we compare $\mathrm{PRG}_{1}$ with the well-known Gennaro's generator 8] in the setting of concrete security. For both generators, we determine parameters (e.g., the size of the seed) such that a desired level of provable security is reached, while minimizing the computational effort per output bit.

Security of Gennaro's generator is based on a variant of the discrete logarithm problem, that is, the discrete logarithm with short exponent (DLSE) problem. Let $x, y$ be elements of a multiplicative group $\mathbb{G}$. The $c$-DLSE problem is to find $s, 0 \leq s<2^{c}$, such that $y=x^{s}$ given $x, y$ and the parameter $c$. Clearly, the DLSE problem is not harder to solve than the original discrete logarithm problem.

Now, we recall the basic results of 8 .

Let $g$ be a generator of $\mathbb{Z}_{p}^{*}$, where $p$ is an $n$-bit safe prime. For a nonnegative integer $x$ let $\ell_{j}(x) \in\{0,1\}$ denote the $j$-th least significant bit of $x$ :

$$
x=\sum_{j} \ell_{j}(x) 2^{j-1} .
$$

Let $x_{1} \in R \mathbb{Z}_{p-1}$ be the seed. Gennaro's generator (Algorithm 3) transforms the seed into the pseudorandom sequence of length $k(n-c-1)$.

The following statement is the concrete version of Theorem 2 of [8].

Theorem 3 (Gennaro). Suppose Gennaro's pseudorandom generator is not $(T, \epsilon)$-secure. Then there exists an algorithm that solves the $c$-DLSE in $\mathbb{Z}_{p}^{*}$ in time $8 c(\ln c)(k / \epsilon)^{3} T$ with probability $1 / 2$.

Gennaro's generator outputs $(n-c-1)$ bits per modular exponentiation with $c$-bit exponent. The standard right-to-left exponentiation costs on average $c / 2$ multiplications and $c$ squarings. Assume that a squaring modulo $p$ takes about $80 \%$ of the time of a multiplication modulo $p$ (cf. [18]). Then, the average computational effort is $1.3 c n^{2} /(24 \cdot 360(n-c-1))$ time units per output bit. Our goal is now to determine $n$ and $c$ that minimize the computational effort under 


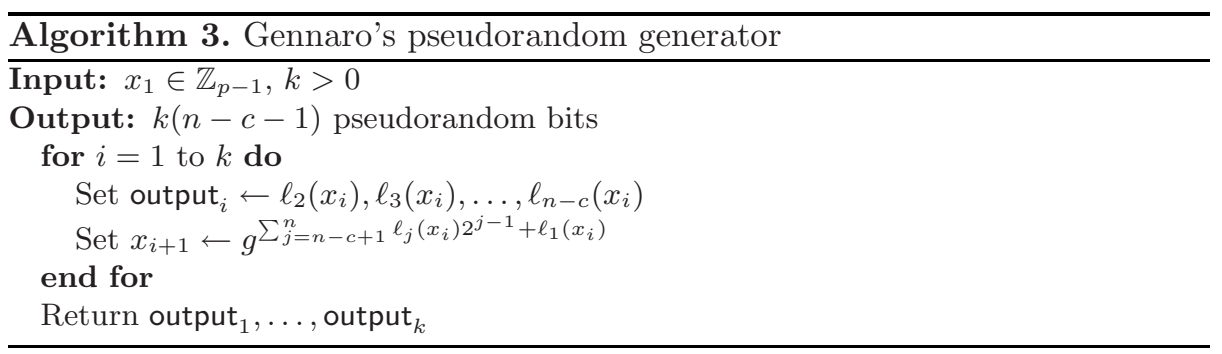

the condition that the generator is $(T, \epsilon)$-secure for all $T, \epsilon$ satisfying $T / \epsilon<2^{80}$ with a natural limitation $T \geq 1$ time unit.

Theorem 3 implies that Gennaro's generator is $(T, \epsilon)$-secure if

$$
16 c(\ln c)(k / \epsilon)^{3} T<T_{D L S E}\left(\mathbb{Z}_{p}^{*}\right),
$$

where $T_{D L S E}\left(\mathbb{Z}_{p}^{*}\right)$ is the running time of the fastest algorithm for solving the $c$-DLSE problem in $\mathbb{Z}_{p}^{*}$. The fastest algorithms for solving the DLSE problem are the discrete logarithm variant of the NFS and the Pollard's lambda method. The complexity of the latter is close to $2 \cdot 2^{c / 2}$ multiplications in $\mathbb{Z}_{p}^{*}$, that is, $2^{c / 2+1} n^{2} /(24 \cdot 360)$ time units (cf. [26]). Note that $k=M /(n-c-1)$, where $M$ is the total number of pseudorandom bits produced by the generator. Thus, Gennaro's generator is $(T, \epsilon)$-secure if

$$
\frac{16 c(\ln c) M^{3} T}{\epsilon^{3}(n-c-1)^{3}}<\min \left[L(n), 2^{c / 2+1} n^{2} /(24 \cdot 360)\right] .
$$

For $M=2^{20}, T / \epsilon<2^{80}$ with a natural limitation $T \geq 1$ the optimal parameters are $n \approx 18000, c \approx 520$.

The secure length of the modulus turns out to be quite large. Recall that generator $\mathrm{PRG}_{1}$ is provably secure for much smaller parameter $n$, namely, $n \approx$ 1600. The reason is that the reduction in Theorem 3 is not tight in the sense that a distinguisher for Gennaro's generator is transformed into the far less efficient solver for the DLSE problem (note that $\epsilon$ is raised to the power of 3 in the statement of Theorem 3). On the contrary, the reduction in Theorem 2 is much tighter.

To compare Gennaro's generator with generator $\mathrm{PRG}_{1}$, we determine the computational effort for both generators.

1. The average computational effort of Gennaro's generator is $1.3 \mathrm{cn}^{2} /(24$. $360(n-c-1))$ time units per output bit. For $n=18000, c=520$, we get about 1500 time units per output bit.

2. The generator $\mathrm{PRG}_{1}$ outputs $n$ bits at the cost of 2 modular exponentiations with $n$-bit exponent. The average computational effort for $n=1600$ is $2.6 n^{2} /(24 \cdot 360) \approx 770$ time units per output bit.

Thus, for $M=2^{20}$ bits to be produced and for the level of security of $2^{80}$ time units, generator $P R G_{1}$ is about 2 times faster than Gennaro's generator. 
Furthermore, the seed length of generator $\mathrm{PRG}_{1}$ is more than 10 times shorter (1600 bits versus 18000 bits).

We draw the attention of the reader to the way the comparison is done. At first sight, it seems that Gennaro's generator is more efficient than generator $\mathrm{PRG}_{1}$ since Gennaro's generator outputs almost $n$ bits per modular exponentiation with a short $c$-bit exponent, while generator $\mathrm{PRG}_{1}$ outputs $n$ bits per 2 exponentiations with a full-size exponent. However, it should not be neglected that the $n$ 's in these two cases are different. Due to the tighter reduction, generator $\mathrm{PRG}_{1}$ is provably secure for much smaller $n$. This is the main reason why generator $\mathrm{PRG}_{1}$ turns out to be more efficient for the same level of security.

\section{Concluding Remarks and Open Problems}

Independent of our work, Jiang recently proposed a pseudorandom generator which is also provably secure under the DDH assumption [14. The security properties of Jiang's generator are similar to ours (hence his generator compares similarly to Gennaro's generator). On the other hand, in comparison with our construction Jiang's generator has two major disadvantages. Firstly, Jiang's generator can be based only on the group of quadratic residues modulo a safe prime while our construction extends to many other groups of prime order. Secondly, the seed of our generator $\mathrm{PRG}_{1}$ is twice as short as the seed of Jiang's generator.

The seed length is a critical issue for pseudorandom generators. For instance, if a pseudorandom generator is used as a keystream generator for a stream cipher the seed length corresponds to the length of the secret key. Also, from a theoretical point of view, the seed length is perhaps the most important parameter of a pseudorandom generator, as discussed in detail in the recent paper by Haitner et al. 12 .

In this respect, we make the following observation. The seed of Jiang's generator can be reduced in length by a factor of two, making it as short as the seed of our generator $\mathrm{PRG}_{1}$, provided one assumes the intractability of the so-called square decisional Diffie-Hellman problem (see, e.g., 31]). The modification to Jiang's generator is to update the state $A_{t}$ as follows: set $A_{t}=g^{\left|A_{t-1}\right|_{p}^{2}}$, using the notation of [14], rather than setting $A_{t}=g^{\left|A_{t-2}\right|_{p}\left|A_{t-1}\right|_{p}}$. Note, however, that the square decisional Diffie-Hellman problem has not been studied as extensively as the standard DDH problem.

Finally, we note that constructing an efficient provably secure pseudorandom generator based on the intractability of the DDH problem on an ordinary elliptic curve is an interesting open problem. For most ordinary elliptic curves, the best known methods for solving the elliptic curve discrete logarithm problem are the exponential square root attacks, so to reach a security level of $2^{80}$ time units it suffices to let the size of the group be about 160 bits. Hence, such an elliptic curve based generator would allow for a considerable reduction of the seed length, potentially to a seed of 160 bits only.

To implement the DDH generator based on an elliptic curve, one has to construct an efficiently computable function that bijectively maps the points of the 
curve to $\mathbb{Z}_{q}$, where $q$ is the order of the group. This function seems to be difficult to construct for ordinary elliptic curves. For some supersingular elliptic curves, the function can be constructed (see, e.g., [16]). However, the latter curves cannot be used for the DDH generator since the DDH problem in these curves can be easily solved by computing Weil pairings [23].

\section{Acknowledgements}

We thank David Galindo for fruitful discussions. We also thank the anonymous referee for pointing us to the paper of Jiang [14.

\section{References}

1. E. Barker and J. Kelsey, Recommendation for random number generation using deterministic random bit generators, December 2005, NIST Special Publication (SP) 800-90.

2. M. Blum and S. Micali, How to generate cryptographically strong sequences of pseudo-random bits, SIAM Journal on Computing 13 (1984), no. 4, 850-864.

3. D. Brown, Conjectured security of the ANSI-NIST Elliptic Curve RNG, Cryptology ePrint Archive, Report 2006/117, 2006, http://eprint.iacr.org/

4. O. Chevassut, P. Fouque, P. Gaudry, and D. Pointcheval, The Twist-AUgmented Technique for Key Exchange, Public Key Cryptography-PKC 2006, Lecture Notes in Computer Science, vol. 3958, Springer-Verlag, 2006, pp. 410-426.

5. R. Cramer and V. Shoup, Design and analysis of practical public-key encryption schemes secure against adaptive chosen ciphertext attack, SIAM Journal on Computing (2003), 167-226.

6. R. Fischlin and C. P. Schnorr, Stronger security proofs for RSA and Rabin bits, Journal of Cryptology 13 (2000), no. 2, 221-244.

7. P. Fouque, D. Pointcheval, J. Stern, and S. Zimmer, Hardness of distinguishing the $M S B$ or LSB of secret keys in Diffie-Hellman schemes, ICALP (2), 2006, pp. 240 251.

8. R. Gennaro, An improved pseudo-random generator based on the discrete logarithm problem, Journal of Cryptology 18 (2005), no. 2, 91-110.

9. R. Gennaro, H. Krawczyk, and T. Rabin, Secure hashed Diffie-Hellman over non-DDH groups, Cryptology ePrint Archive, Report 2004/099, 2004, http://eprint.iacr.org/,

10. K. Gjøsteen, Comments on Dual-EC-DRBG/NIST SP 800-90, Draft December 2005, March 2006, http://www.math.ntnu.no/ kristiag/drafts/ dual-ec-drbg-comments.pdf

11. O. Goldreich, Foundations of cryptography, Cambridge University Press, Cambridge, UK, 2001.

12. I. Haitner, D. Harnik, and O. Reingold, On the power of the randomized iterate, Advances in Cryptology - Crypto 2006, Lecture Notes in Computer Science, vol. 4117, Springer-Verlag, 2006, pp. 22-40.

13. J. Håstad, R. Impagliazzo, L. A. Levin, and M. Luby, Construction of a pseudorandom generator from any one-way function, SIAM Journal on Computing 28 (1999), 1364-1396. 
14. S. Jiang, Efficient primitives from exponentiation in $\mathbb{Z}_{p}$, ACISP, Lecture Notes in Computer Science, vol. 4058, Springer-Verlag, 2006, pp. 259-270.

15. A. Juels, M. Jakobsson, E. Shriver, and B. K. Hillyer, How to turn loaded dice into fair coins, IEEE Transactions on Information Theory 46 (2000), no. 3, 911-921.

16. B. S. Kaliski, Elliptic curves and cryptography: A pseudorandom bit generator and other tools, Ph.D. thesis, MIT, Cambridge, MA, USA, 1988.

17. D. E. Knuth, Seminumerical algorithms, third ed., vol. 3, Addison-Wesley, Reading, MA, USA, 1997.

18. A. K. Lenstra and E. R. Verheul, The XTR public key system, Advances in Cryptology_Crypto 2000, Lecture Notes in Computer Science, vol. 1880, SpringerVerlag, 2000, pp. 1-19.

19. _ـ Selecting cryptographic key sizes, Journal of Cryptology 14 (2001), no. 4, $255-293$.

20. M. Luby, Pseudorandomness and cryptographic applications, Princeton University Press, Princeton, NJ, USA, 1994.

21. U. M. Maurer, Towards the equivalence of breaking the Diffie-Hellman protocol and computing discrete algorithms, CRYPTO, 1994, pp. 271-281.

22. U. M. Maurer and S. Wolf, Diffie-Hellman oracles, CRYPTO, 1996, pp. 268-282.

23. A. Menezes, T. Okamoto, and S. A. Vanstone, Reducing elliptic curve logarithms to logarithms in a finite field, IEEE Transactions on Information Theory 39 (1993), no. 5, 1639-1646.

24. M. Naor and O. Reingold, Number-theoretic constructions of efficient pseudorandom functions, Journal of the ACM 51 (2004), no. 2, 231-262.

25. S. Patel and G. S. Sundaram, An efficient discrete log pseudo random generator, CRYPTO, 1998, pp. 304-317.

26. J. M. Pollard, Kangaroos, monopoly and discrete logarithms, Journal of Cryptology 13 (2000), no. 4, 437-447.

27. B. Schoenmakers and A. Sidorenko, Cryptanalysis of the Dual Elliptic Curve pseudorandom generator, Cryptology ePrint Archive, Report 2006/190, 2006, http://eprint.iacr.org/,

28. R. Shaltiel, Recent developments in explicit constructions of extractors., Bulletin of the EATCS 77 (2002), 67-95.

29. R. Steinfeld, J. Pieprzyk, and H. Wang, On the provable security of an efficient RSA-based pseudorandom generator, Cryptology ePrint Archive, Report 2006/206, 2006, http://eprint.iacr.org/

30. S. Wolf, Information-theoretically and computationally secure key agreement in cryptography, Ph.D. thesis, ETH Zurich, 1999.

31. F. Zhang, R. Safavi-Naini, and W. Susilo, An efficient signature scheme from bilinear pairings and its applications, Public Key Cryptography 2004, Lecture Notes in Computer Science, vol. 2947, Springer-Verlag, 2004, pp. 277-290. 